\title{
De nouveaux procédés d'extraction des huiles pour des produits finis de haute qualité
}

\author{
Michel PARMENTIER \\ Sandrine GUILLEMIN \\ Reine BARBAR \\ Michel LINDER \\ Jacques FANNI \\ Laboratoire de science et génie alimentaires, \\ ENSAIA, INPL, \\ 2 avenue de la Forêt de Haye, F-54500 \\ Vandoeuvre les Nancy \\ $<$ Michel.Parmentier@ensaia.inpl-nancy.fr>
}

\begin{abstract}
New concerns have appeared recently from the European and American consumers about quality, safety and environmental impact of the food products. In the particular case of European countries, the coming back of traditional products with a high sentimental value should be noticed. To fit the forthcoming demand of the consumer, the Oils and Fats industry have to develop a new approach based on alternative technologies that may emerge from the research with very specific objectives: no solvents, mild technologies, less energy consumption and final wastes. The purpose of this article is to discuss about the new biological tools capable to generate alternative ways for the oil elaboration: use of enzymes for destructuration of the vegetal or animal tissues and the subsequent utilisation of membrane techniques for the separation and purification steps. These new technologies involving less heat and mechanical stress to the oil, lead to a better "native" quality of the final products. However, fundamental research is needed on the separation mechanisms of those complex mixtures to optimise the process and prepare the transfer to the industrial conditions. The present article reports a contribution on the aqueous extraction of rapeseed oil as an example
\end{abstract}

Key words: extraction, final products, quality

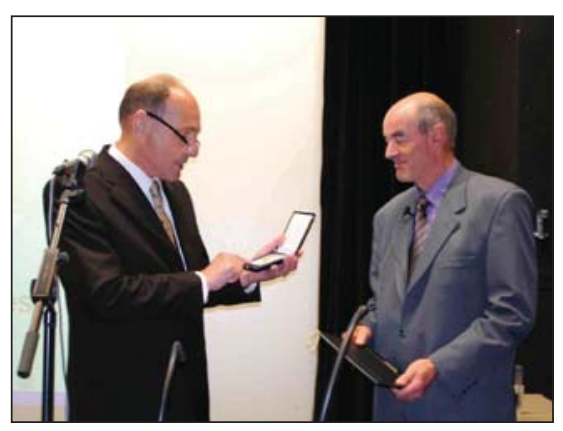

Photo 1. Remise de la médaille Normann à M. Parmentier par F. Spener au nom de la DGF.

lisation, décoloration et désodorisation) fait appel à une succession de process thermiques et mécaniques qui ne sont pas sans effet sur les molécules grasses et les composés mineurs nutritionnellement intéressants: ils altèrent donc la qualité originelle du produit, du moins en ce qui concerne quelques molécules-clés comme les anti-oxydants, les vitamines liposolubles...

Le consommateur possède aujourd'hui dans ce domaine une référence : I'huile d'olive vierge, dont l'image de pureté naturelle constitue l'un des arguments principaux de vente. Son succès n'est pas un hasard, car c'est le produit qui le premier dans cette filière a su imposer une image de pureté et de naturalité et surfer sur la vague d'exigences nouvelles du public en termes de traditionalité des produits et de respect des qualités natives et des images fortes véhiculées par quelques faiseurs d'opinion [3].

Quelle peut être la réponse des technologues à ce type de demande dans le cas des huiles concurrentes et, en particulier, les huiles de graines qui constituent la base de la consommation de corps gras visibles dans nos sociétés? L'analyse de la demande sociétale concernant les produits peut se développer en deux points, l'un concerne le respect de l'environnement et l'autre une moindre utilisation de la chaleur, tout particulièrement des hautes températures qui dégradent plus fortement les molécules sensibles.

La première de ces demandes conduit à étudier les alternatives à l'extraction par solvant organique, donc le remplacement de l'épuisement à I'hexane. Même si l'absence d'alternative crédible conduit actuellement à continuer ce type de traitement, tous les technologues savent que l'extraction à l'hexane est condamnée à terme. On ne peut plus aux États-Unis construire de nouvel atelier sur ce modèle. En revanche - il faut bien assurer la production - on peut faire des extensions d'unités existantes, avec des cahiers des charges extrêmement coercitifs, aussi bien en termes de résidus dans le produit fini que dans les quantités libérées dans l'environnement, domaine dans lequel des progrès significatifs ont été et sont toujours 
réalisés. La recherche de solvants alternatifs moins toxiques (pour l'homme et pour l'environnement) n'ayant pas donné de résultats convaincants, il faut bien se tourner vers des solutions plus radicalement différentes.

La deuxième demande sociétale nécessite de soumettre les matières premières à moins de stress thermique, ce qui conduit forcément à remettre en cause certaines phases du raffinage, ce qui n'est de toute façon pas simple. En effet, on ne peut supprimer des étapes à haut stress thermique comme le blanchiment ou la désodorisation, que dans la mesure où le procédé d'extraction aura permis d'éliminer les composés indésirables et les résidus phytosanitaires.

C'est donc de tout le process d'extractionraffinage qu'il convient de parler si l'on veut progresser dans ce domaine [4]. Le débat global sur ces questions a été largement développé dans les communautés scientifiques et techniques actives sur les procédés lipidiques. Les uns comme les autres ont admis que la recherche devait se tourner vers des procédés moins drastiques (Mild Refining, Environmental Friendly Processing...) [5].

En pratique, les solutions ne sont pas nombreuses. II n'existe que deux types d'outils utilisables pour résoudre ce challenge. Sans solvant, l'extraction par pression donne des rendements en huile trop faibles pour être économiquement rentable. Par ailleurs, le procédé d'extraction doit assurer une bonne purification de l'huile produite, afin de ne nécessiter ensuite qu'un raffinage doux. II faut donc d'abord déstructurer le tissu végétal adipeux pour libérer les huiles, et ceci peut être réalisé à I'aide d'enzymes. II faut ensuite mettre en œuvre les séparations qui permettent de sélectionner les composants désirables et non désirables dans l'huile comestible. Les techniques membranaires peuvent y aider.

\section{Les bases de l'extraction aqueuse des huiles de graines}

L'extraction sans solvant de l'huile de colza a été développée à l'échelle de petites unités décentralisées, fonctionnant par pression à froid, tout particulièrement en Allemagne. Quelque 200 unités de ce type, traitant de 0,5 à 20 tonnes de graines par jour, ont été répertoriées en RFA [6]. Ce procédé par pression à froid permet de ne mettre en œuvre ni solvant, ni enzyme, ni raffinage, à l'exception d'un simple lavage et de protocoles drastiques de préparation des graines et de stockage de l'huile. Toutefois, les auteurs indiquent une forte proportion de ces huiles qui ne satisfont pas aux critères réglementaires de qualité des huiles de consommation humaine.

Les procédés comparés d'extraction de l'huile de colza sont présentés en figure 1.

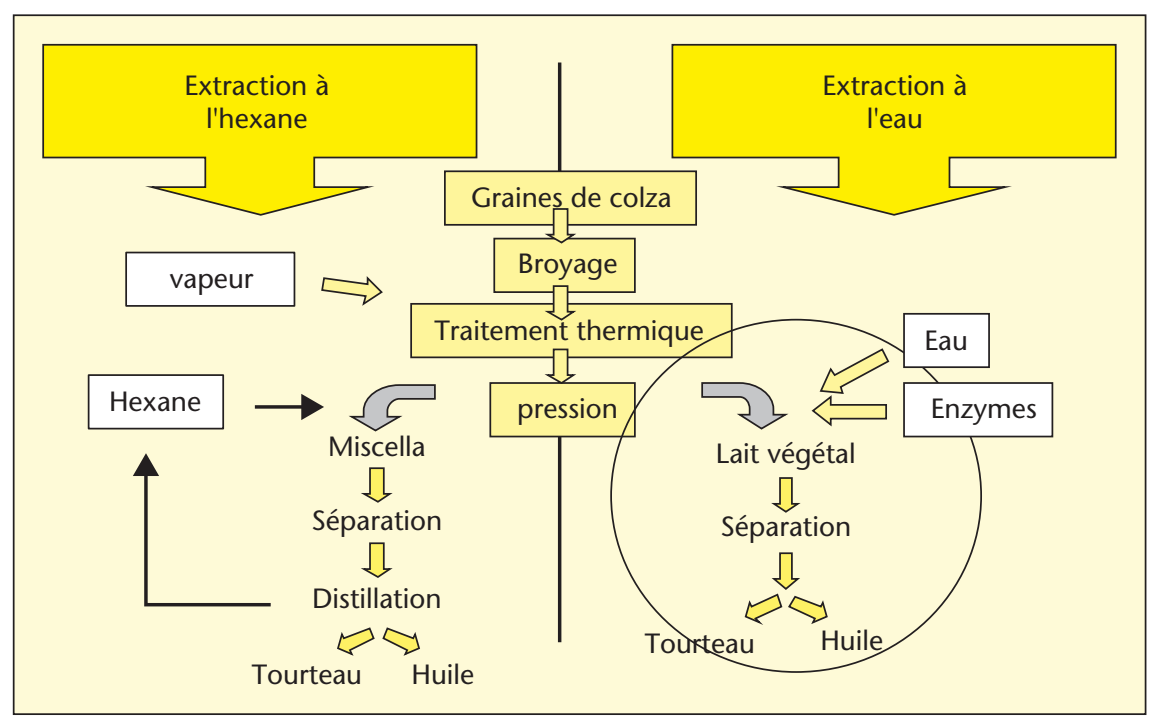

Figure 1. Procédés comparés d'extraction à l'hexane et à l'eau.

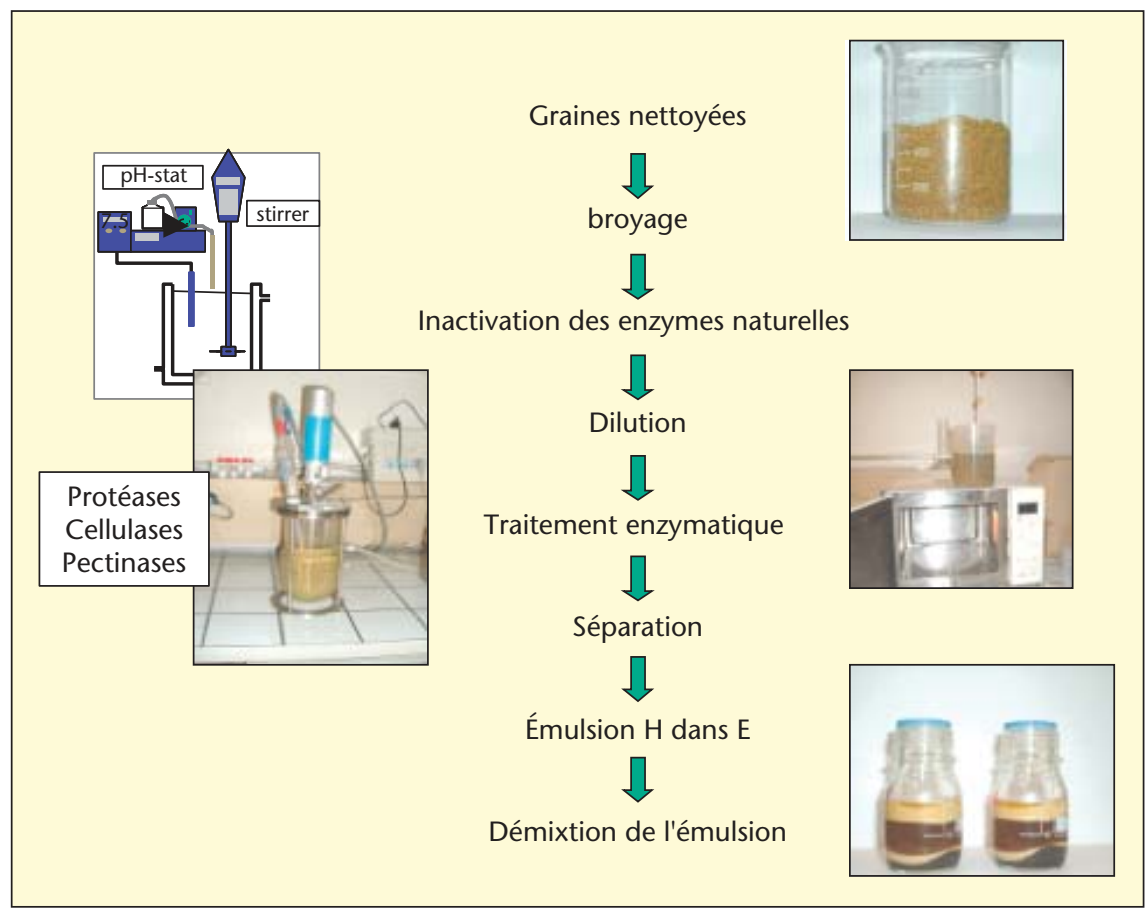

Figure 2. Procédés d'extraction aqueuse d'huile de colza

Après une séquence commune de préparation des graines, d'inactivation des activités enzymatiques naturelles et de pressage, les deux procédés divergent: d'un côté l'extraction à I'hexane, et de l'autre, l'action des enzymes de déstructuration, puis les étapes de séparation.

Un certain nombre d'enzymes peuvent être mises en œuvre pour déstructurer le tissu végétal. Le détail du procédé d'extraction aqueuse mis en œuvre ici est montré dans la figure 2.
Les enzymes de paroi, cellulases et pectinases sont utilisables dans ce sens. Leur action de destruction des parois et des tissus végétaux peut être complétée par celle des protéases, qui vont agir plus finement au niveau des sousensembles intracellulaires constitués par les associations huile neutre-lipides polairesprotéines-eau. Le paramétrage exact de la succession des actions enzymatiques est évidemment complexe, car toutes les configurations 
d'actions successives de ces molécules influent sur l'extraction finale de l'huile. Dans tous les cas, la quantité d'huile directement récupérable par décantation au-dessus du milieu réactionnel dépend:

- de la déstructuration mécanothermique de la graine avant l'action des enzymes. Le broyage, sa finesse, les chocs thermiques, tout ce qui permet la dilacération est influent sur la suite du procédé ;

- de l'ordre de mise en œuvre des différentes enzymes. En effet les enzymes de parois ont pour résultat la dégradation des parois végétales, ce qui est une étape indispensable pour permettre l'action de la deuxième famille d'enzymes, les protéases. Ces dernières ont pour effet majeur de déstabiliser le complexe naturellement stable constitué par l'association des triglycérides, des phospholipides, des protéines et de l'eau, tout ceci à l'intérieur des cellules;

- des conditions physicochimiques qui prévalent lors de la séparation : température, $\mathrm{pH}$, dilution.

La centrifugation permet de mettre en évidence l'effet du traitement enzymatique et la diminution de la stabilité du système complexe évoqué ci-dessus. Si tel est le cas, une centrifugation fournit 4 phases, comme l'indique la figure 3.

À partir d'un milieu réactionnel qui décante très difficilement, à l'exception d'une légère pellicule d'huile neutre à la surface, une centrifugation à $5000 \mathrm{~g}$ pendant 10 minutes sépare de façon très nette les différentes phases en fonction de leur densité, c'est-à-dire du bas (phase la plus dense) au haut de la charge (phase la moins dense), on distingue: les coques (phase I), magma sombre très bien séparé de la phase protéique lourde insoluble (phase II), puis de I'hydrolysat protéique soluble dans la phase aqueuse (phase III). Entre la

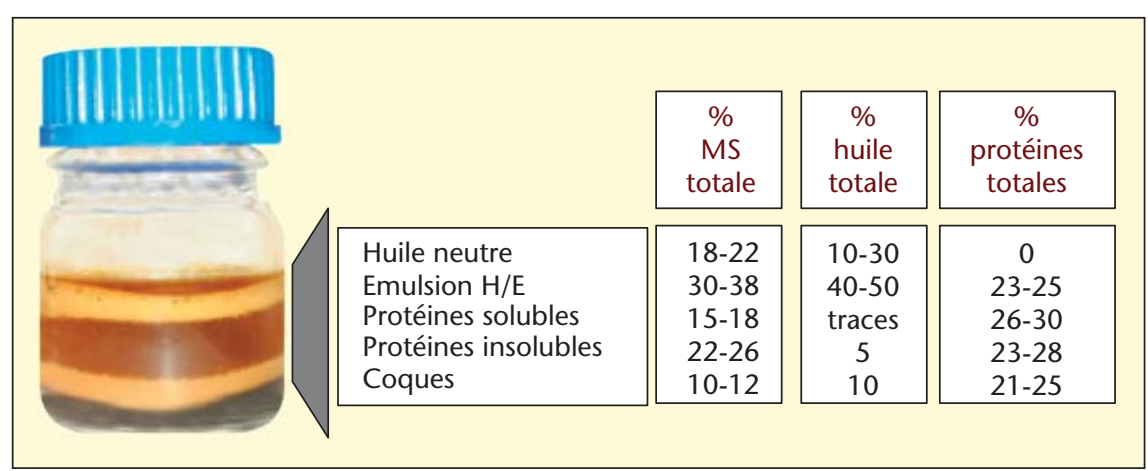

Figure 4. Répartition de la matière sèche, de l'huile et des protéines dans les différentes phases centrifugées après extraction à l'eau des graines de colza.

phase légère constituée $d$ 'huile neutre (phase V) directement libérée et la phase aqueuse, apparaît une couche émulsionnée (phase IV). Cette couche est une émulsion blanche d'huile dans l'eau, stabilisée par les phospholipides et les fragments protéiques.

Ce n'est pas la première fois que nous observons ce type de ségrégation de phases après action des enzymes de déstructuration des tissus adipeux. Des situations similaires ont été obtenues lors de traitements de produits animaux, comme les œufs et les co-produits de poisson par exemple (travaux confidentiels, non publiés).

Un point important à ce stade de l'extraction est de connaître précisément la répartition de I'huile dans les phases I à V, dont dépend le rendement final en huile, facteur déterminant d'une industrialisation. Ce bilan a été établi dans la figure 4, qui reprend phase par phase la répartition de la matière sèche, des protéines et de l'huile.

Les résultats sont exprimés en $\%$ de la matière (sèche, huile, protéines) totale. Ils font apparaître que $85 \%$ de l'huile est présente dans les

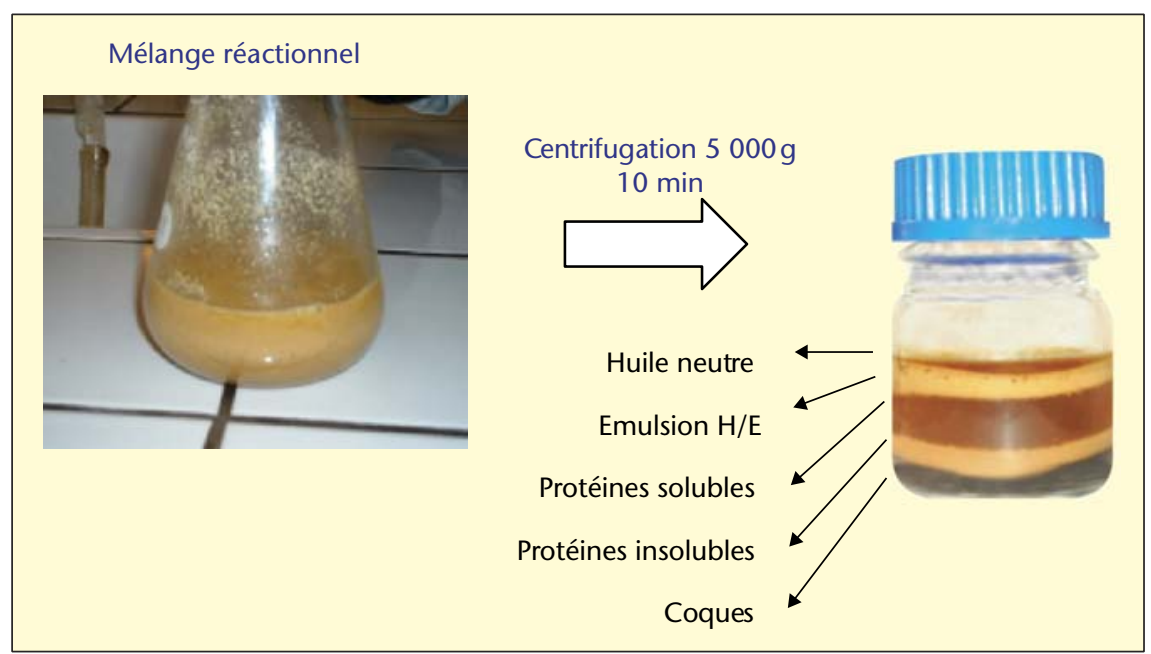

Figure 3. Fractionnement du mélange réactionnel après extraction à l'eau sur des graines de colza. phases IV et V. Les $15 \%$ restantes sont adsorbées sur les solides (phases II et I), qui constitueront par la suite la partie directement valorisable en nutrition animale. En revanche, les protéines sont quasiment uniformément réparties entre les différentes phases, à l'exception de l'huile surnageante. Une attention particulière doit être apportée à la phase émulsionnée: elle contient environ $50 \%$ de I'huile totale, probablement la majeure partie des phospholipides, et un quart des protéines. C'est évidemment une configuration extrêmement stable, ce qui complique largement le problème de la récupération de cette huile, qui représente environ la moitié de la quantité totale présente dans la graine initiale.

Le problème de la déstabilisation de l'émulsion de la phase IV constitue donc l'un des verrous de ce procédé d'extraction aqueuse. La centrifugation n'apporte pas de réponse satisfaisante, c'est pourquoi nous avons testé des solutions membranaires.

Déstabiliser une émulsion par séparation membranaire n'est pas un problème simple. II n'existe d'ailleurs pas à l'heure actuelle de dispositif technique, membranaire ou non, capable de fournir d'un côté la phase aqueuse et de l'autre la phase grasse à partir d'une émulsion stable. Ce n'est que dans certains cas particuliers que la solubilisation de l'azote protéique avant filtration permet d'obtenir, sur des membranes hydrophiles, la séparation huile/eau. $C^{\prime}$ est le cas par exemple avec le babeurre, où des facteurs de concentration de l'ordre de 10 fois ont été obtenus par Fanni et al. [7]. Toutefois, il n'y a pas dans ce cas d'obtention d'une phase «huile» isolée, mais seulement d'un enrichissement en huile du rétentat.

L'originalité de notre nouvelle approche membranaire réside dans la constitution d'une cellule associant deux valves hydrophilehydrophobe conçues sur le modèle de la figure 5 et montées tête-bêche. Ces valves sont construites par dépôt sur une base poreuse d'un composé polaire induisant une orientation 


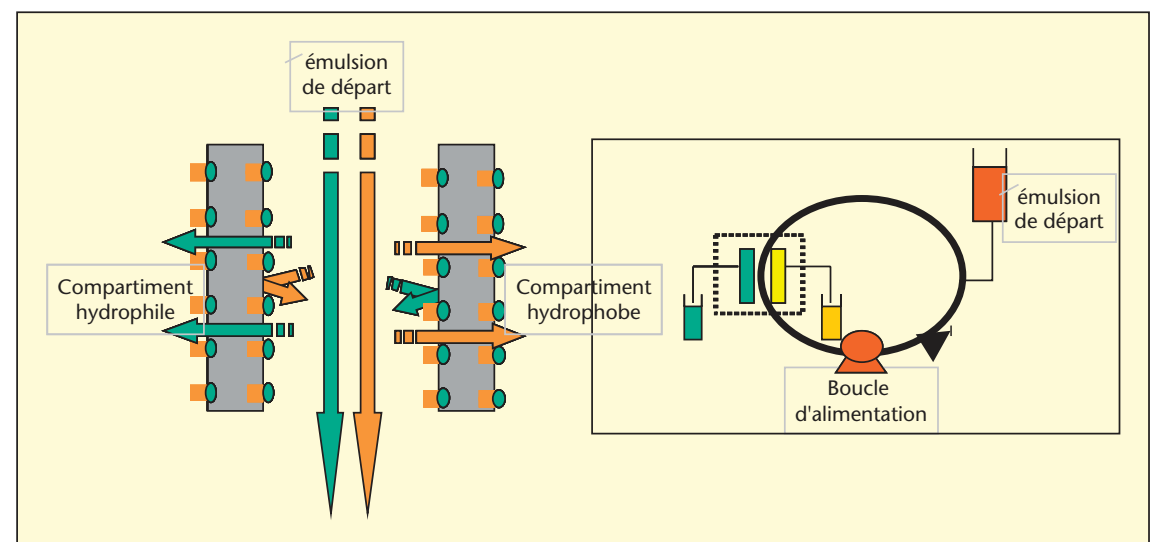

Figure 5. Cellule de séparation membranaire construite par l'association de deux valves hydrophobe/hydrophile montées en «tête-bèche ".

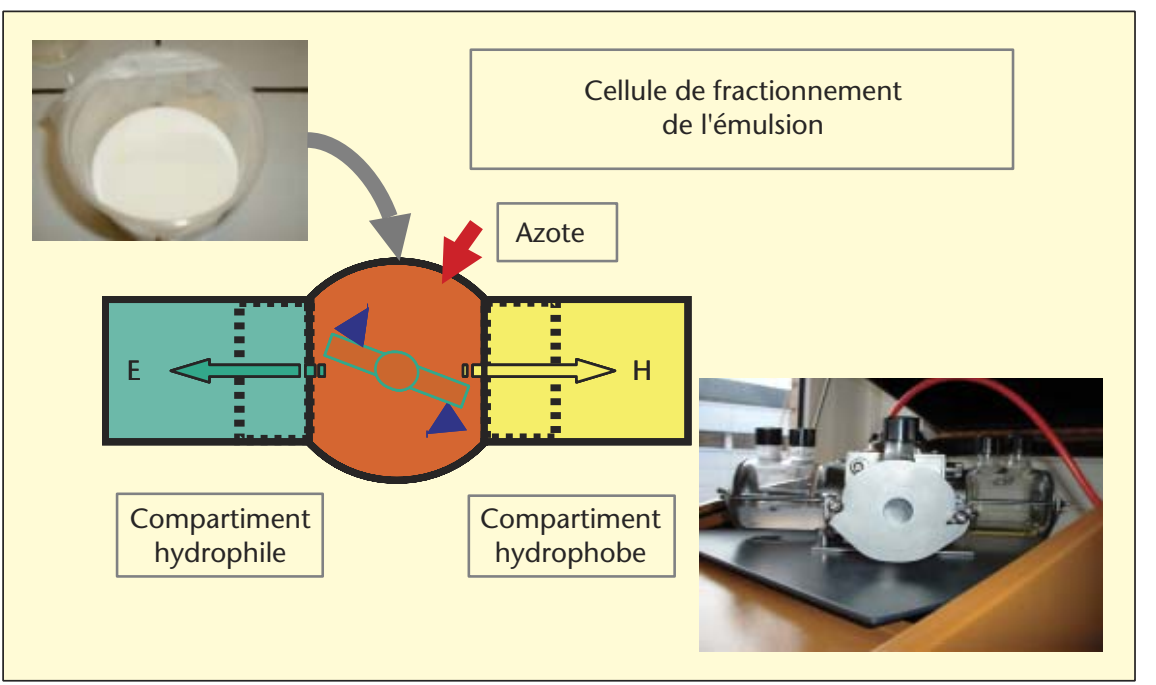

Figure 6. Séparation d'une émulsion huile de maïs-eau-SDS par membrane.

moléculaire à la surface, de telle sorte que la membrane ainsi générée fonctionne comme une jonction en électronique : les molécules compatibles avec la surface peuvent traverser, mais dans un seul sens.

Le montage de deux valves tête-bêche permet en théorie de séparer les composants d'un milieu mixte (ou émulsion), les composés hydrophiles passant dans le compartiment hydrophile alors que les molécules hydrophobes traversent l'autre valve vers le compartiment hydrophobe.

Le seul exemple d'approche similaire a été proposé par Kurentjes et al. en 1992 [8] pour s'approchant du modèle : des membranes globalement hydrophiles pour le compartiment hydrophile et des membranes hydrophobes pour le compartiment hydrophobe.

La cellule est alimentée par une émulsion huile de maïs-eau-SDS. Le résultat est visible sur la figure 6: la partie aqueuse s'écoule dans le compartiment hydrophile, et I'huile dans l'autre. II s'agit d'un résultat tout à fait encourageant, même si l'émulsion qui a été démixée n'est pas aussi stable que celle obtenue dans l'extraction aqueuse d'une huile de graine. Pour ce qui concerne l'émulsion de la phase IV les résultats sont encore décevants, le système apparaissant encore trop stable pour permettre la séparation des deux familles de constituants. La recherche se poursuit donc dans deux directions complémentaires : déstabiliser l'émulsion en éliminant tout ou partie des composés amphiphiles présents d'une part, et d'autre part, travailler sur le matériau membranaire afin de le rendre plus sélectif en termes de balance hydrophile-lipophile.

NB. Cette recherche est supportée par ONIDOL, 12 rue Georges V, Paris.

\section{RÉFÉRENCES}

1. KARLESKIND. Manuel des corps gras. Lavoisier, Paris.

2. HAMM W. HAMILTON RJ Edible oil processing. Sheffield, UK : Sheffield Academic Press, 2000.

3. DILAS G. Images et réalité de I'huile d'olive pour le consommateur européen. OCL 2004 ; 11(3) : 199-202.

4. KOVARI K. Chevreul Medal lecture. Recent developments, new trends in seed crushing and oil refining. 3rd Euro Fed Lipid Congress, 2004, Edinburgh, Scotland.

5. TEN BRINK HB, VAN DUIJN G. Refined seed oil production, a milder future? Inform 2003; 14(5) : 274-5. membrane hydrophile/membrane hydrophobe en série, la première produisant un perméat hydrophile (eau + savons), alors que I'autre achève la séparation en produisant un perméat d'huile neutre hydrophobe. Les deux montages sont toutefois très différents.

La principale difficulté de ce type de montage réside dans les membranes elles-mêmes, étant entendu qu'il n'existe pas d'offre de membranes de ce type dans le commerce. II faut donc les construire. Ce travail est en cours, et s'avère ardu. C'est pourquoi des essais ont été conduits avec des membranes commerciales
6. MATTHAÜS B, BRÜHL L. Cold-pressed edible rapeseed oil production in Germany. Inform $2004 ; 15(4)$ : 266-8.

7. FANNI I, BENNEWITZ D, LINDER M, PARMENTIER M. Enzymatic-catalysed enrichment of phospholipids in buttermilk : Optimisation of reaction conditions. DGF \& AFECC Joint Congress. Germany : Würzburg, 2000.

8. KEURENTJES JTF, SLUIIS JTM, FRANSSEN RJH, VAN'T RIET K. Extraction and fractionation of fatty acids from oil using a ultrafiltration membrane. Ind Engng Chem Res 1992 ; 31 : 581-7. 